\title{
ON ABELIAN PERMUTATION GROUPS
}

\author{
R. Bercov and L. Moser \\ (received January 20, 1965)
}

The principal object of this note is to determine the maximal order of Abelian subgroups of the symmetric group $S_{n}$ of degree n. We also discuss some related results and problems.

THEOREM 1. A largest Abelian subgroup of $\mathrm{S}_{n}$ has order $f(n)$ where

$$
\begin{array}{ll}
f(n)=3^{m} & \text { if } n=3 m \\
f(n)=4 \cdot 3^{m-1} & \text { if } n=3 m+1 \\
f(n)=2.3^{m} & \text { if } n=3 m+2 .
\end{array}
$$

An alternative formulation is that $f(n)$ is the order of a largest Abelian permutation group of degree $\leq n$.

In a recent issue of this Bulletin, A. Evans posed the problem of showing that the above $f(n)$ is the largest number which is a product of positive integers whose sum is $n$. The solution by V. Linis appeared in [1]. The same problem was posed earlier by L. Moser and a solution by L. Carlitz appeared in [2]. The result also appears in [3] and, one would guess, in earlier work as well.

Let $g(n)$ be the order of a largest Abelian subgroup of $S_{n}$. We first use the weIl known fact that a transitive Abelian group is regular (i.e. has the same order and degree) [4] to show that $g(n) \leq f(n)$. Let $|S|$ denote the number of elements of a set $S$, and Iet $G$ be an Abelian permutation group on a set $\Omega$ with $|\Omega|=n$. We denote the image of $\alpha \in \Omega$ under $x \in G$ by

Canad. Math. Bull. vol. 8, no. 5, October 1965 
$\alpha^{\mathrm{x}}$, and $\left\{\alpha^{\mathrm{x}} \mid \mathrm{x} \in \mathrm{G}\right\}$ by $\alpha^{\mathrm{G}}$. The distinct $\alpha^{\mathrm{G}}$ are then the orbits of $G$, which partition $\Omega$. We denote them by $\mathrm{T}_{1}, \ldots, \mathrm{T}_{\mathrm{k}}$. The $\mathrm{i}^{\text {th }}$ transitive constituent $\mathrm{G}_{i}$ of $\mathrm{G}$ is $\left\{x_{i} \mid x \in G\right\}$ where $x_{i}$ is defined by

$$
\begin{aligned}
& \alpha^{\mathrm{x}_{\mathrm{i}}}=\alpha^{\mathrm{x}} \quad \text { for } \quad \alpha \in \mathrm{T}_{\mathrm{i}} \\
& \alpha^{\mathrm{x}_{\mathrm{i}}}=\alpha \text { for } \alpha \notin \mathrm{T}_{\mathrm{i}} .
\end{aligned}
$$

$G_{i}$ is transitive and Abelian, hence regular; thus $\left|G_{i}\right|=\left|T_{i}\right|$. Since $x$ is the permutation product of the permutations $x_{i}$, we have $G \leq G_{1} \ldots G_{k}$; thus $|G| \leq\left|G_{1} \ldots G_{k}\right|$. But $\mathrm{k}$ $\left|G_{1} \ldots G_{k}\right|=\prod_{i=1}\left|G_{i}\right|$, since for $i \neq j, G_{i}$ fixes every $\mathrm{k}$ $\alpha \in \Omega$ which $G_{j}$ moves. We therefore have $|G| \leq \prod_{i=1}\left|G_{i}\right|$ $=\prod_{i=1}^{k}\left|T_{i}\right| \leq f(n)$ since $\sum_{i=1}^{k}\left|T_{i}\right|=n$ because the orbits of $G$ partition $\Omega$. Maximizing over $|G|$ we obtain $g(n) \leq f(n)$ as required.

That $g(n)>f(n)$ holds is easily seen. We partition $n$ into $\mathrm{k} k$ positive parts $m_{1}, \ldots, m_{k}$ with $\sum_{i=1} m_{i}=n$ and $\underset{i=1}{\prod_{i}} m_{i}=f(n)$. The cycles on $\mathrm{m}_{1}, \ldots, \mathrm{m}_{\mathrm{k}}$ distinct symbols generate cyclic groups of order $m_{1}, \ldots, m_{k}$ whose product is an Abelian group of order $f(n)$ and degree $\leq n$.

Thus the proof of theorem 1 is complete.

We are indebted to Professor J.S. W. Wong for raising with us the problem of determining $g(n)$ and to Professor W. R. Scott for drawing our attention to the following closely related results of O. Ore [5] and A. Powsner [6]: 
THEOREM 2. The smallest degree of a faithful permutation representation of an Abelian group is the sum of its invariants.

It is amusing to note that the function $f(n)$ arises also in the following graph theoretic problem: What is the largest number $c(n)$ such that there exists an ordinary graph of $n$ vertices and $c(n)$ cliques? (A clique is a set of vertices every pair of which are joined by an edge such that no vertex outside the set is joined to all of them.) That $c(n)=f(n)$ was conjectured by P. Erdös and L. Moser and independent proofs (unpublished) have been found by P. Erdös and by J. W. Moon and L. Moser.

Returning to group theory, we remark that the problem of determining the order $h(n)$ of a largest cyclic subgroup of $S_{n}$ has received considerable attention. It is trivial that $h(n)$ is the largest number expressible as the least common multiple of positive integers whose sum is $n$ [7]. Here, it is too much to expect a simple explicit formula for $h(n)$, but E. Landau [8] proved

\section{THEOREM 3. $\log h(n) \sim \sqrt{n \log n}$}

The fact that $h(52)=180,180$ and its grave implications for card players is discussed by W. W. Rouse BaII [9]. More recently $h(n)$ has been studied by $S$. W. Golomb [3] who tabulated $h(n)$ for $n<120$ and made a number of observations concerning $h(n)$ including a disproof by counterexample of the conjecture that if $n=p_{1}+\ldots+p_{k}\left(p_{i}\right.$ the $i^{\text {th }}$ prime) then $h(n)=p_{1} \cdots p_{k}$.

Since permutations correspond to permutation matrices, theorem 1 gives the maximum number of $\mathrm{n} \times \mathrm{n}(0,1)$ matrices in the class of permutation matrices which commute in pairs. One could ask more generally for the maximum number of $(0,1)$ matrices in other classes which commute in pairs. The following classes might be worth studying from this point of view:

(i) all $\mathrm{n} \times \mathrm{n}(0,1)$ matrices

(ii) the "mapping into" $\mathrm{n} \times \mathrm{n}(0,1)$ matrices, namely those with a single 1 in each row.

(iii) the $\mathrm{n} \times \mathrm{n}$ graph matrices. These are characterized by $a_{i j}=a_{j i}$ and $a_{i i}=0$. 
(iv) the $\mathrm{n} \times \mathrm{n}$ round-robin tournament matrices. These are characterized by $a_{i j}+a_{j i}=1$ for $i \neq j$ and $a_{i i}=0$.

\section{REFERENCES}

1. A. Evans, Problem 80, solution by V. Linis. Can. Math. Bull. 7 (1964), p. 626.

2. L. Moser, Problem 125, solution by L. Carlitz. $\pi \mu \epsilon$ Journal, 3 (1961), pp. 232-233.

3. S. W. Golomb, Two combinatorial problems of mathematical and practical significance. Mimeographed notes (1960). $11 \mathrm{pp}$. + tables.

4. W. R. Scott, Group Theory. Prentice Hall (1964), p. 265.

5. O. Ore, Contributions to the theory of groups of finite order. Duke Math. Jour. 5 (1939), pp. 431-460.

6. A. Powsner, Über eine Substitutionsgruppe kleinster Gerades die einer gegebener Abelschen Gruppe isomor ph ist. Commun. Instit. Sci. Kharkov 4, 14 (1937), pp. 151-157.

7. W. H. H. Hudson, Educational Times Reprints 2. London (1865), p. 105.

8. E. Landau, Handbuch der Lehre von der Verteilung der Primzahlen. Verlag von B. G. Teubner. Leipzig und Berlin 1909. vol. 1, pp. 222-229.

9. W. W. Rouse Ball, Math Recreations and Essays. Revised by H. S. M. Coxeter. 11th ed. MacMillan and Co. London (1944), pp. 311-312.

University of Alberta 